\title{
Electrodeposited Assembly of Additive-Free Silk Fibroin Coating from Pre-Assembled Nanospheres for Drug Delivery
}

\author{
Xian Cheng, Dongmei Deng, Lili Chen, John A. Jansen, Sander G. C. Leeuwenburgh, and Fang Yang*
}

Cite This: ACS Appl. Mater. Interfaces 2020, 12, 12018-12029

Read Online

ABSTRACT: Electrophoretically deposited (EPD) polymerbased coatings have been extensively reported as reservoirs in medical devices for delivery of therapeutic agents, but control over drug release remains a challenge. Here, a simple but uncommon assembly strategy for EPD polymer coatings was proposed to improve drug release without introducing any additives except the EPD matrix polymer precursor. The added value of the proposed strategy was demonstrated by developing a novel EPD silk fibroin (SF) coating assembled from pre-assembled SF nanospheres for an application model, that is, preventing infections around percutaneous orthopedic implants via local delivery of antibiotics. The EPD mechanism of this nanosphere coating involved oxidation of

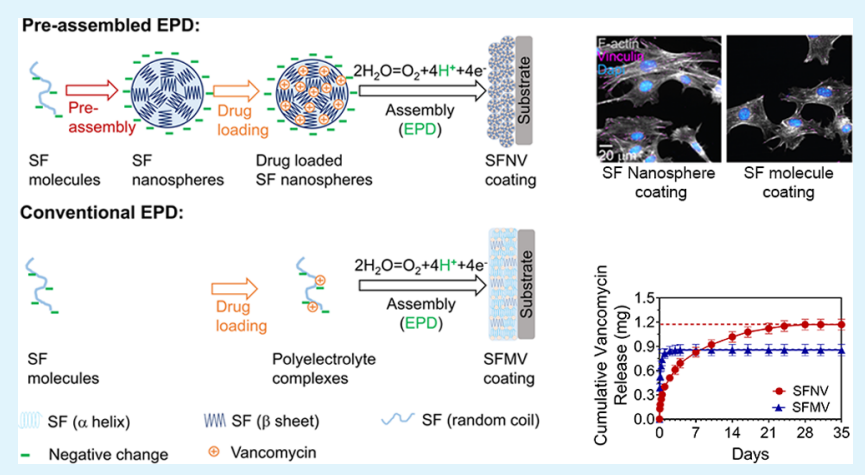
water near the substrate to neutralize SF nanospheres, resulting in irreversible deposition. The deposition process and mass could be easily controlled using the applied EPD parameters. In comparison with the EPD SF coating assembled in a conventional way (directly obtained from SF molecule solutions), this novel coating had a similar adhesion strength but exhibited a more hydrophobic nanotopography to induce better fibroblastic response. Moreover, the use of nanospheres as building blocks enabled 1.38 and 21 times enhancement on the antibiotic release amount and time (of 95\% maximum dug release), respectively, while retaining drug effectiveness and showing undetectable cytotoxicity. This unexpected release kinetics was found attributable to the electrostatic and hydrophobic interactions between the drug and nanospheres and a negligible initial dissolution effect on the nanosphere coating. These results illustrate the promising potential of the pre-assembled strategy on EPD polymer coatings for superior control over drug delivery.

KEYWORDS: coating, pre-assembly, drug delivery, electrodeposition, silk fibroin

\section{INTRODUCTION}

Polymer-based coatings are widely used on the surface of different medical devices, such as drug-eluting stents, ${ }^{1,2}$ orthopedic (or dental or otorhinolaryngologic) fixators, and implants, ${ }^{3,4}$ to act as reservoirs for sustained release of therapeutic and signaling agents. Compared with other commonly used coating methods, such as plasma spraying, one of the most common methods in the industry today, electrophoretic deposition (EPD) is a solution-based and binder-free technique, which is very suitable to construct homogeneous polymer coatings with high purity and finetunable thickness on complex geometries, porous structures, and nonline-of-sight surfaces that medical devices usually possess. ${ }^{5}$ EPD has been extensively reported to prepare a wide range of (sensitive) polymer coatings for biomedical applications ${ }^{6}$ in a mild aqueous environment at room (or low) temperature, with simple equipment requirements, ${ }^{7}$ short processing time, ${ }^{8}$ high preparation efficiency, ${ }^{9}$ as well as upscaling potential for commercial production. ${ }^{10}$

In the past, the conventional way to functionalize EPD coatings with therapeutic drugs is to mix the drug into matrix polymer precursor solution before deposition of the mixture, ${ }^{11}$ but control over release kinetics is often not achieved because of the poor affinity and interaction between the drug and coating matrix. ${ }^{5}$ Many recent studies aimed at introducing a variety of other material-based drug delivery vehicles into the coating matrix polymer ${ }^{9,12-14}$ (e.g., carbon nanotubes or gelatin nanoparticles in a chitosan matrix coating) or using a cross-linking agent such as genipin ${ }^{15}$ between the drug and matrix to improve control over the drug release of EPD coatings.

Although introducing additional chemicals to currently existent material systems is frequently applied in research laboratories to modify material functions, it is not a preferred solution from a point of view of clinical application and commercial production that the introduced chemicals might

Received: December 2, 2019

Accepted: February 10, 2020

Published: February 10, 2020 
often increase the complexity and cost of manufacture and raise the uncertainty of biosafety. ${ }^{16}$ For EPD coatings, it should be noted that the matrix polymer precursors themselves can be assembled into a variety of nanostructures to regulate drug release. ${ }^{17}$ Therefore, we hypothesized that the drug release capability of the EPD polymer coating can be enhanced via assembling structures from pre-assembled nanoarchitectures, without introducing any new chemicals except the polymer precursor of the coating matrix itself.

To demonstrate the added value of this assembly strategy on EPD polymer coatings, a novel silk fibroin (SF) EPD coating assembled from pre-assembled SF nanospheres, instead of being assembled from SF molecules used in the conventional EPD assembly strategy, is developed to improve the delivery drug in an application model, that is, preventing infections around percutaneous orthopedic implants. Although antibioticloaded EPD coatings have been applied on various orthopedic implants to prevent infections, ${ }^{11,12,18-20}$ the controlled and sustained release of the antibiotics and their long-term effects remain to be a challenge. Compared to other widely used EPD polymer precursors, such as chitosan, SF has recently attracted a lot of research attention to be used for EPD coatings ${ }^{5,21-23}$ for biomolecule drug delivery because of its stabilization effect on sensitive biological compounds (e.g., antibiotics), ${ }^{24}$ excellent biocompatibility, ${ }^{25}$ and hypoallergenicity. ${ }^{26}$ Moreover, SF molecules themselves can be easily assembled into SF nanospheres, ${ }^{27}$ attractive vehicles for sustained delivery of manifold drugs from antibiotics and anticancer drugs to growth factors. $^{28}$

In this study, SF nanospheres were first pre-assembled by precipitation reaction. To reveal the assembly mechanisms of nanosphere coating, we investigated the $\zeta$-potential and particle size of SF nanospheres as a function of $\mathrm{pH}$, and the coating thickness as a function of EPD processing parameters. In comparison with the conventional EPD SF coatings directly assembled from SF molecules (SFM coating), this nanosphere coating (SFN coating) was characterized in terms of conformation changes, topography, wettability, degradation, adhesion strength, and fibroblastic response. Then, drug release profiles of SFN and SFM coatings were compared, and the underlying mechanisms of superior control over drug release of SFN coating were investigated. These results not only make great progress and improvement for the EPD SF coatings simply by assembling the coatings from pre-assembled nanoarchitectures but also indicate the potential of the preassembly strategy on EPD polymer coating field as a simple, additive-free, cost-effective approach to achieve superior control over drug release.

\section{MATERIALS AND METHODS}

2.1. Preparation of SF Solution. SF solution was prepared as previously described. ${ }^{29}$ Briefly, Bombyx mori silk cocoons provided by Prof. Aichun Zhao (State Key Laboratory of Silkworm Genome Biology, Southwest University, Chongqing, P. R. China) were first degummed in boiled $0.02 \mathrm{M} \mathrm{Na}_{2} \mathrm{CO}_{3}$ solution for $30 \mathrm{~min}$ and then rinsed with Milli- $\mathrm{Q}$ water. After drying, the extracted silk was dissolved in $9.3 \mathrm{M} \mathrm{LiBr}$ at $60{ }^{\circ} \mathrm{C}$ for $4 \mathrm{~h}$ and then dialyzed with Milli$\mathrm{Q}$ water using the dialysis membrane $\left(M_{\mathrm{W}}=3500\right)$. Insoluble residues were removed by centrifugation at $5000 \mathrm{rpm}$ for $1 \mathrm{~h}$ at $4{ }^{\circ} \mathrm{C}$. Finally, the SF concentration in aqueous solution was adjusted to $8 \mathrm{wt} \%$.

2.2. Preparation of SF Nanosphere Suspension. SF nanospheres were synthesized via a precipitation reaction, by which SF aqueous solution ( $5 \mathrm{wt} \%$ ) was dropwise added into five times-volume acetone, as previously reported. ${ }^{30}$ Then, SF nanospheres were washed with Milli-Q water and centrifuged at $5000 \mathrm{rpm}$ for $1 \mathrm{~h}$ for three times to remove acetone. SF nanosphere dispersions were obtained following sonication of nanospheres in Milli-Q water for $5 \mathrm{~min}$ at $100 \%$ amplitude, and pulse rate of $1 \mathrm{~s}$ on, $1 \mathrm{~s}$ off, using a sonicator (Emerson Industrial Automation, Branson Europe, Dietzenbach, Germany). The morphology of nanospheres was measured by scanning electron microscopy (SEM) (Sigma-300, Zeiss, Germany).

2.3. Characterization of SF Nanosphere Suspension. The $\zeta$ potential and particle size of SF nanospheres were measured using dynamic light scattering (DLS, Zetasizer, Nano-S, Malvern Instruments Ltd., U.K.) by dispersion of nanospheres in HEPES buffer (5 $\mathrm{mM}$ ) with adjusted $\mathrm{pH}$ (using $\mathrm{HCl}$ or $\mathrm{NaOH}$ ). Each value represents an average of three measurements.

2.4. Preparation of Substrates. Commercially pure titanium disks (grade 2, Baoji Titanium Industry, China) were utilized as a deposition substrate. To obtain a smooth surface, the Ti plates were manually ground with Grit 600 and Grit 2500 grinding paper (Struers, the Netherlands). ${ }^{31}$ Then, they were ultrasonically (VWR, the Netherlands) cleaned in acetone, ethanol, and water for $5 \mathrm{~min}$ of each treatment, and finally subjected to argon plasma glow discharge (Radio frequency glow discharge machine, Harrick Scientific Corp., U.S.A.) for $5 \mathrm{~min}$. Rough surfaces were prepared by sandblasting with $0.25-0.50 \mathrm{~mm}$ grit. Subsequently, these discs were acid-etched with $\mathrm{HCl} / \mathrm{H}_{2} \mathrm{SO}_{4}$ for $30 \mathrm{~min}$ at $60{ }^{\circ} \mathrm{C}^{32}$ and then also ultrasonically cleaned with acetone, ethanol, water, and glow discharge. Smooth 316L stainless steel and CoCrMo (Tiger International BioMetals Co., Ltd., China) with a similar treatment were also used as substrates to demonstrate the feasibility of constructing SFN coatings on different typical medical metallic surfaces.

2.5. EPD of SFN Coating. Pretreated metal substrates were used as a working electrode, and a parallel pure titanium disk of the same size and shape was used as the counter electrode. For each experiment, fresh $10 \mathrm{~mL}$ of SFN dispersion was used. The distance between the positive and negative electrodes was $10 \mathrm{~mm}$. To investigate the EPD parametric control over SFN coating deposition, the EPD process was first carried out by connecting both electrodes to a direct current power supply (model 6614C, Agilent Technologies) with different concentrations from 0.5 to $1.75 \mathrm{wt} \%$ at a constant electric field of $5 \mathrm{~V} / \mathrm{cm}$ for $2 \mathrm{~min}$. In another set of experiments, the same time of $2 \mathrm{~min}$ and $1 \mathrm{wt} \%$ SFN suspension were applied for different electric fields $(3-8 \mathrm{~V} / \mathrm{cm})$. Finally, different deposition time (1-10 min) was applied with $1 \mathrm{wt} \%$ SFN suspension at a constant electric field of $5 \mathrm{~V} / \mathrm{cm}$. During deposition, the SFN suspensions were stirred using a magnetic stirrer $(50 \mathrm{rpm})$. For following material characterization and biological assessment SFN coating, SFN coating was deposited at $5 \mathrm{~V} / \mathrm{cm}$ for $2 \mathrm{~min}$ from $1.0 \mathrm{wt} \% \mathrm{SF}$ nanosphere suspension. After the deposition, the $\mathrm{Ti}$ disks were carefully withdrawn from the solution, rinsed with Milli- $Q$ water three times, and slowly air-dried in a box to prevent coating cracks. Finally, the samples were cross-linked by water vapor annealing in a vacuum desiccator overnight at room temperature.

2.6. EPD of SFM Coating. For comparison, SFM coatings were directly assembled form SF molecule solution as the conventional EPD method. Similarly, a fresh $10 \mathrm{~mL}$ SF molecule solution was used. The distance between the positive and negative electrodes was 10 $\mathrm{mm}$. EPD was carried out by connecting both electrodes to a direct current power supply with a concentration of $1 \mathrm{wt} \%$ at a constant electric field of $5 \mathrm{~V} / \mathrm{cm}$ for $2 \mathrm{~min}$. After the deposition, the disks were carefully withdrawn from the solution, rinsed with Milli- $Q$ water for three times, and slowly dried in a box. Finally, they were cross-linked by water vapor annealing in a vacuum desiccator overnight at room temperature.

2.7. Material Characterization of Coatings. The topography of the coating surfaces was examined by SEM (Sigma-300, Zeiss, Germany). Fourier-transform infrared spectroscopy (FTIR) analysis of the coatings was performed by attenuated total reflectance infrared spectroscopy (UATR two, PerkinElmer, the Netherlands). The roughness and thickness of the coatings were measured using the Profilometer (ProScan, U.S.A.) $(n=3)$ as the method previous reported. ${ }^{33}$ The wettability of different SF surfaces was determined by 
detecting the static water contact angles of the surfaces with an Optical Tensiometer (Theta Lite, Biolin Scientific, Sweden) $(n=3)$. For the degradation test, coatings were immersed in $1.5 \mathrm{~mL}$ of PBS at $37^{\circ} \mathrm{C}$ for 14 days, and PBS solutions were replaced freshly every $24 \mathrm{~h}$ $(n=3)$. At specified time points, the samples were rinsed via agitation in Milli-Q water for 5 times (each for $2 \mathrm{~min}$ ) and dehydrated in an oven at $60{ }^{\circ} \mathrm{C}$ overnight. Immediately following the removal from the oven, the samples were weighed.

The adhesion strength of the coatings was tested using a standard lap shear tensile test (Supporting Information, Figure S1a). ${ }^{31}$ Each test specimen was an assembly of a coated substrate and a matching substrate sample with the same dimension bonded together using a thin layer of epoxy adhesive (Loctite 415, USA). The samples were held by grips of a Universal Testing Machine (MTS Systems, 858 mini bionix II, U.S.A.). The pull test was run at a constant cross-head displacement of $0.50 \mathrm{~mm} / \mathrm{min}$ until failure $(n=3)$. The results were included only when the sample failed at the coating/substrate interface, as shown by the exposure of the metallic substrate $(n=3$, Supporting Information, Figure S1b).

2.8. Biological Assessment of Coatings. NIH-3T3 (ATCC, USA), a mouse fibroblastic cell line, was cultured in Dulbecco's modified essential medium (Gibco, Invitrogen Corp., Paisley, Scotland) supplemented with $10 \%$ calf serum (Gibco, Invitrogen Corp., Paisley, Scotland) at $37{ }^{\circ} \mathrm{C}$ in a humidified $5 \% \mathrm{CO}_{2}$ atmosphere. Coated disks were sterilized by UV light, placed in 24well tissue culture plates, immersed in cell culture media for $4 \mathrm{~h}$, and then seeded at a density of 5000 cells $\cdot \mathrm{cm}^{-2}$, while cell culture coverslips (CS, Thermo Fisher, U.S.A.) were used as a positive control.

Cells cultured on the SFM and SFN coating surfaces after $24 \mathrm{~h}$ were washed with PBS and then fixed with 2 wt \% glutaraldehyde in $0.1 \mathrm{M}$ sodium-cacodylate solution for $20 \mathrm{~min}$. Subsequently, cells were dehydrated in a graded series of ethanol (70, 80, 90, 96, and $100 \%$ ethanol for $5 \mathrm{~min}$ each), followed by observing the morphology of the cells by SEM. For immunofluorescent studies, cells were fixed using $4 \%$ paraformaldehyde for $10 \mathrm{~min}$, followed by PBS washing for three times, and then permeated with $0.1 \%$ Triton X-100 for $10 \mathrm{~min}$. Subsequently, the cells were blocked with $1 \%$ BSA for $30 \mathrm{~min}$ and incubated with the anti-vinculin antibody (1:200, ab129002, Abcam, U.S.A.) for $1 \mathrm{~h}$. The Alexa Fluor 647-conjugated goat anti-rabbit secondary antibody (1:500, ab150083, Abcam, U.S.A.) was incubated for $1 \mathrm{~h}$. TRITC-phalloidin (1:2000, P1951, Sigma, U.S.A.) was applied to label F-actin, and DAPI (1:2500, D9542, Sigma) was used to mark cell nuclei. Images of stained samples were captured by fluorescent microscopy (Axio Imager Microscope Z1, Zeiss, Germany).

All the images were analyzed by Image J (NIH, USA). To measure focal adhesions (FAs) area per cell, the grayscale vinculin image was thresholded to produce a black and white image from which the pixels representing FAs were counted and summed, following a step-by-step quantitative $\mathrm{FA}$ analysis protocol as previously reported. ${ }^{34}$ To measure the cell area and perimeter, the cellular morphology were determined using the thresholding method from F-actin fluorescent images, and then, they were used to calculate the cell area and cell perimeter. The cell shape index (CSI) was calculated using the formula as previously reported ${ }^{35}$

$$
\text { CSI }=\frac{4 \pi \times \text { cell area }}{(\text { cell perimeter })^{2}}
$$

where a line and a circle have CSI values of 0 and 1 , respectively.

The RNA was extracted using an RNeasy Mini Kit (Qiagen, U.S.A.), according to the manual instruction and reversed to cDNA using TaqMan Reverse Transcription kit (Bio-Rad, U.S.A.). Subsequently, cDNA was added with a Fast SYBR Green Master Mix Kit and complemented by the PRISM 7500 sequence amplification system (Applied Biosystems, USA). Vinculin was tested with GAPDH as the housekeeping gene. The primer sets of genes are listed in Table S1 (Supporting Information). The mRNA levels of target genes were normalized by the level of GAPDH mRNA and calculated via the $2^{-\Delta \Delta C_{t}}$ method.

The total cell number was quantified at specific time intervals $(1,3$, and 7 days) using a cell counting kit (CCK-8; Dojindo, Japan). The result of CCK-8 was tested spectrophotometrically (Bio-Tek FL600 microplate fluorescence reader, Biotek, U.S.A.) according to the manufacturer instructions. Three samples per group were tested.

After $24 \mathrm{~h}$ of incubation, lactate dehydrogenase (LDH) Assay kit (Thermo Fisher Scientific, U.S.A.) was used for determining cytocompatibility of drug-loaded coatings by measuring the LDH activity released from damaged cells. Cell culture coverslips without and with 5\% DMSO were used as positive and negative controls, respectively, while wells added with $2 \%$ (v/v) Triton-X100 and only culture medium were used as the high and low controls, respectively. The results from the LDH assay were tested spectrophotometrically according to the manufacturer instructions. Three samples per group were tested. The cytocompatibility was calculated using the formula as previously reported ${ }^{12}$

$$
\begin{aligned}
& \text { Cytocompatibility (\%) } \\
& \qquad=\left(1-\frac{\text { experimental value }- \text { low control }}{\text { high control }- \text { low control }}\right) \times 100 \%
\end{aligned}
$$

2.9. Detection on Vancomycin Concentration. Concentration of vancomycin in this study was detected by HPLC using a Hitachi HPLC machine, which consisted of a Hitachi L-2130 pump, a Hitachi L-2400 UV detector, a Hitachi L-2200 auto sampler, and a LiChrospher RP-18 end-capped HPLC column $(125 \mathrm{~mm} \times 4 \mathrm{~mm}$, particle size $5 \mu \mathrm{m}$ ). The mobile phase consisted of $50 \mathrm{mM}$ ammonia phosphate buffer ( $\mathrm{pH} 3$, adjusted with $\left.\mathrm{H}_{3} \mathrm{PO}\right)$; acetonitrile (90/10 $\mathrm{v} / \mathrm{v})$ was used for the detection of vancomycin. The supernatant (30 $\mu \mathrm{m})$ containing vancomycin was injected with a flow rate of $1 \mathrm{~mL} /$ $\mathrm{min}$. Then, the concentration of vancomycin was quantified at 196 $\mathrm{nm}$.

2.10. Optimization of the Vancomycin Loading Amount. Various weight ratios of vancomycin and SF nanospheres were used for determining the optimum loading capacity for SF nanospheres. First, different concentrations $(0.8,1.6,2.4,3.2$, and $4 \mathrm{mg} / \mathrm{mL})$ of 5 $\mathrm{mL}$ vancomycin solutions were added into $5 \mathrm{~mL}$ of SF nanosphere suspensions $(20 \mathrm{mg} / \mathrm{mL})$ to reach different weight ratios $(0.04,0.08$, $0.12,0.16$, and 0.20$)$ under stirring for $24 \mathrm{~h}$. Then, vancomycinloaded SF nanospheres were collected by washing with Milli-Q water and centrifugation at $15000 \mathrm{rpm}$ for $5 \mathrm{~min}$ three times to remove any unbound drug. The amount of the unbound drug in the washed supernatant was measured by HPLC. The loading capacity and encapsulation efficiency of vancomycin in SF nanospheres were measured using the following equations

$$
\begin{aligned}
& \text { Loading capacity (\%) } \\
& =\frac{\text { total amount of drug }- \text { amount of unbounded drug }}{\text { weight of SFNs }} \\
& \times 100 \% \\
& \text { Encapsulation efficiency (\%) } \\
& =\frac{\text { total amount of drug }- \text { amount of unbounded drug }}{\text { total amount of drug }} \\
& \times 100 \%
\end{aligned}
$$

2.11. EPD of Vancomycin-Loaded Coatings. After optimization, a weight ratio (drug/protein) of 0.12 was chosen to load drug for the following study. For vancomycin-loaded SFN coating (SFNV coating), $5 \mathrm{~mL}$ of vancomycin solution $(2.4 \mathrm{mg} / \mathrm{mL})$ was added into $5 \mathrm{~mL}$ of SF nanosphere suspension $(20 \mathrm{mg} / \mathrm{mL})$ under stirring for 24 h. After the washing and centrifugation step to remove any unbound drug, SFNV was resuspended by $10 \mathrm{~mL}$ Milli- $Q$ water. For vancomycin-loaded SFM coating (SFMV coating), $5 \mathrm{~mL}$ of vancomycin solution $(2.4 \mathrm{mg} / \mathrm{mL})$ was added into $5 \mathrm{~mL}$ of $\mathrm{SF}$ molecule solution $(20 \mathrm{mg} / \mathrm{mL})$ under stirring for $24 \mathrm{~h}$. The SFNV 
a Pre-assembled EPD:
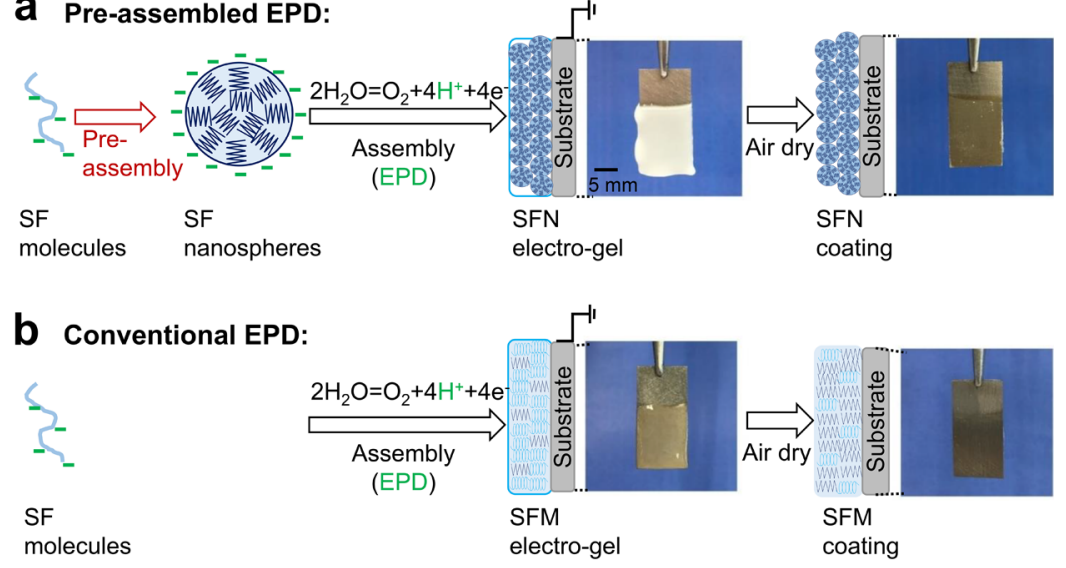

$\sim$ SF (random coil) WM SF ( $\beta$ sheet $) \quad$ SF $(\alpha$ helix $) \quad-$ Negative change
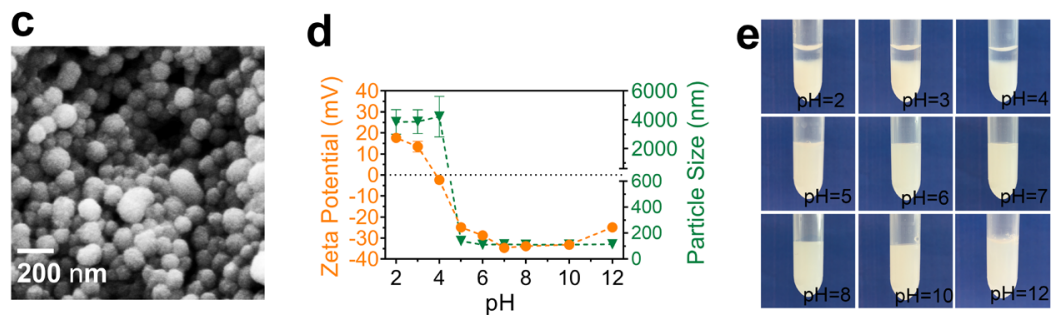

Figure 1. EPD assembly mechanism of SFN coating. (a) Pre-assembly vs (b) conventional assembly of SF EPD coatings. (c) Scanning electron micrographs of SFNs. (d) $\zeta$-Potential and particle size of SFNs as a function of $\mathrm{pH}$. (e) Digital photographs of SFNs showing the stability of SFNs aqueous solution as a function of $\mathrm{pH}$. Error bars represent one standard deviation.

and SFMV coatings were then prepared under the same EPD conditions (electric field of $5 \mathrm{~V} / \mathrm{cm}$ for $2 \mathrm{~min}$ at a total protein concentration of $1 \mathrm{wt} \%)$ as the SFN and SFM coatings used above.

2.12. Drug Release from Coatings. To investigate the release profiles of SFNV and SFMV coatings, the coated disks were placed into glass bottles $(n=3)$, followed by addition of $1 \mathrm{~mL}$ of PBS. To investigate the influence of ionic strength and detergent concentration on the short-term release of drug from SFNV coatings, SFNV coatings were used for an additional release study in media with different (i) ionic strengths $(10,100$, and $1000 \mathrm{mM} \mathrm{NaCl}$ solutions in water) and (ii) detergent concentrations $(0.01,0.1$, and $1 \mathrm{v} / \mathrm{v} \%$ Tween 20$)$. The SFNV coatings were placed into glass bottles $(n=3)$, followed by addition of $1 \mathrm{~mL}$ of the abovementioned media. All the glass bottles were then placed on a shaking plate with a shaking rate of $90 \mathrm{rpm}$ at $37^{\circ} \mathrm{C}$ to perform the release study. At the specific time points, $0.9 \mathrm{~mL}$ of the supernatant was collected and refreshed with the same amount of media. The collected supernatant was stored at $4{ }^{\circ} \mathrm{C}$ until the concentrations of vancomycin were determined using HPLC.

2.13. Antibacterial Efficacy of the Released Drug from Coatings. To investigate the antibacterial efficacy of the released drug from SFNV and SFMV coatings at different time points, both coated disks were placed into glass bottles $(n=3)$, followed by addition of $1 \mathrm{~mL}$ of PBS. All the glass bottles were then placed on a shaking plate with a shaking rate of $90 \mathrm{rpm}$ at $37^{\circ} \mathrm{C}$. At $24 \mathrm{~h}$ before the specific time points, all the PBS was refreshed. At the specific time points, $0.5 \mathrm{~mL}$ of the supernatant was collected and refreshed with the same amount of PBS.

The MICs of each collected supernatant was evaluated using the Staphylococcus aureus ATCC25923 strain. In brief, the overnight culture of $S$. aureus was diluted to $\mathrm{cfu} / \mathrm{mL}$ in two-times concentrated brain heart infusion broth. The SFNV/SFNM supernatants were twofold serial-diluted with PBS. The maximum dilution was 640 -fold. $S$. aureus dilution was mixed with the undiluted or twofold serialdiluted SFNV/SFNM supernatants, PBS and vancomycin solutions at the ratio of $1: 1$, and pipetted into a 96 -well plate $(0.2 \mathrm{~mL} /$ well $)$. The PBS solution was used as a negative control, and the vancomycin solutions at the final concentrations of $0.53,1.06$, and $3.12 \mu \mathrm{g} / \mathrm{mL}$ were used as positive controls. The 96-well plate containing all mixtures was placed in a SpectraMax i3 microplate reader (Molecular Devices, San Jose, CA, USA), and the optical density (OD) value of each well was recorded at the wavelength of $600 \mathrm{~nm}$ at $37^{\circ} \mathrm{C}$ after 10 $h$. In the negative control (PBS) group, the OD value of $S$. aureus culture reached maximum after $10 \mathrm{~h}$. The highest fold of dilution where there was no growth or the growth was below $50 \%$ of those in the negative control group was registered as the dilution times at the break point of the specific SFNV/SFNM supernatant.

2.14. Statistical Analysis. One-way ANOVA was used to determine statistical significance followed by post hoc analysis using the Tukey test. All statistical analyses were performed with GraphPad Prism and Origin software.

\section{RESULTS AND DISCUSSION}

\subsection{Electrodeposited Assembly Mechanism of SFN} Coating. SF molecules were first pre-assembled into SF nanospheres, via precipitation reaction from acetone into which an SF molecule aqueous solution was added dropwise as previously reported. ${ }^{30,36}$ The spherical morphology of the SF nanospheres was confirmed using SEM (Figure 1c). Then, nanospheres were dispersed in water. The $\zeta$-potential and size of nanospheres were examined as a function of $\mathrm{pH}$ via DLS (Figure 1d). The $\mathrm{pH}$ value of SF nanosphere suspension (1 wt $\%)$ was 7.5 , and the average diameter of nanospheres was around $110 \mathrm{~nm}$, which was in agreement with the size observed by SEM and reported previously using this method. ${ }^{36}$ The $\zeta$ potential showed a reversed sigmoidal behavior where the SF nanospheres attained a negative charge of about $-30 \mathrm{mV}$ at $\mathrm{pH}$ values higher than 5 , while the nanospheres had a positive charge of $\sim 18 \mathrm{mV}$ at $\mathrm{pH}$ values below 3 . The inflection point was at $\sim \mathrm{pH} 4$, which is in line with the $\mathrm{pI}$ of SF molecules 

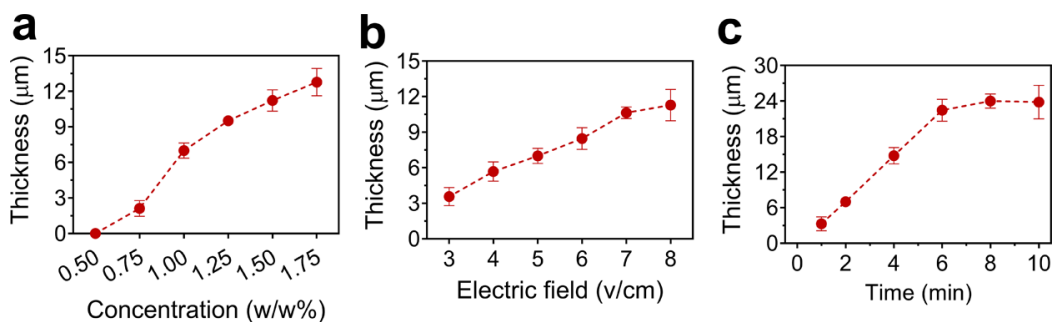

Figure 2. EPD parametric influence on SFN coating thickness. Thickness of the SFN coating as a function of EPD processing parameters, including (a) suspension concentration, (b) electric field, and (c) deposition time. Error bars represent one standard deviation.
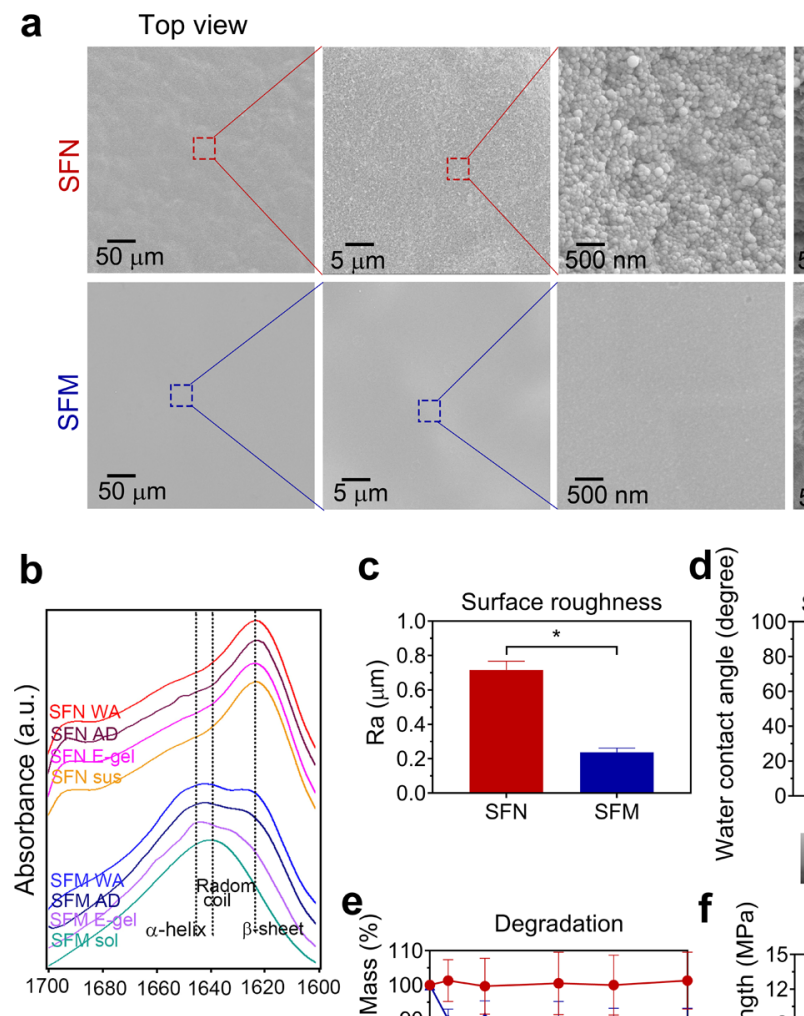

Tilted view
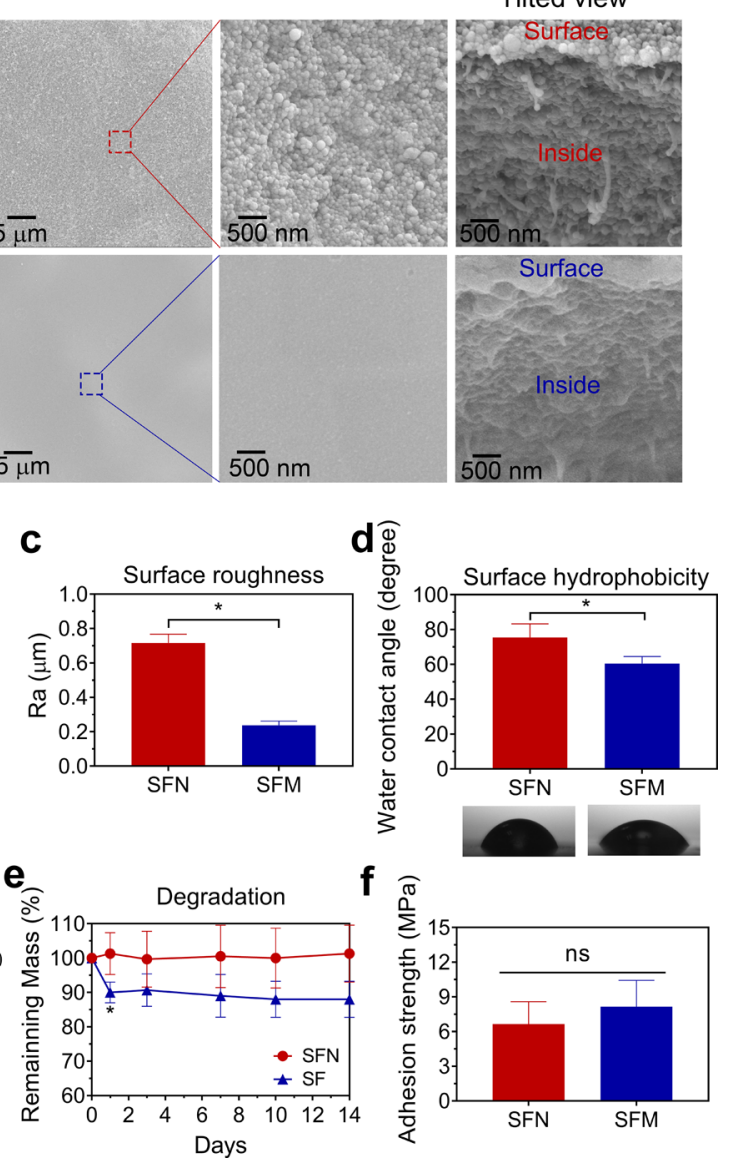

Figure 3. Material characterization SFN coating. (a) Scanning electron micrographs of coatings. (b) FTIR absorbance spectra of the amide I region (between 1695 and $1595 \mathrm{~cm}^{-1}$ ) showing the conformational changes during the preparation process of coatings. WA: after water annealing. AD: after air drying. E-gel: electrogel. sol: solution. sus: suspension. (c) Surface roughness of coatings. (d) Surface wettability determined by water contact angle measurements and representative images of water droplets. (e) Remaining mass of coatings immersed in PBS after 1, 3, 7, and 14 days. (f) Adhesion strength of coatings measured by lap shear tensile testing. Error bars represent one standard deviation $\left({ }^{*} p<0.05\right)$.

$(\sim 4.2) .^{37}$ The inflection point indicates that SF nanosphere dispersions will be stabilized at $\mathrm{pH}>5$ by repulsive interactions between negatively charged SF nanospheres and destabilized at $\mathrm{pH}<4$. This phenomenon also explains our particle size data, which showed a steep increase at $\mathrm{pH}<4$. The digital photographs indicated stable dispersion for SF nanospheres at $\mathrm{pH}>5$, whereas the reduction of $\mathrm{pH}$ yields in phase separation by precipitation (Figure 1e).

Similar to conventional SFM coatings deposited directly from SF molecules solution (Figure 1a), assembling coatings from SF nanosphere suspension was also driven by the applications of a positive potential (Figure 1b). Water was oxidized around the surface of the anode, causing a reduction in $\mathrm{pH}$ in the vicinity of the metal surface (eq 5).

$$
2 \mathrm{H}_{2} \mathrm{O} \rightarrow \mathrm{O}_{2}+4 \mathrm{H}^{+}+4 \mathrm{e}^{-}
$$

The reduction of $\mathrm{pH}$ resulted in protonation of the SF nanosphere surfaces and diminished the repulsive interactions between the nanospheres, thus driving irreversible aggregation and deposition of the nanospheres at the metal surface when the adjacent $\mathrm{pH}$ value arrived at around 4 under appropriate EPD parameters.

3.2. EPD Parametric Influence on SFN Coating Thickness. Then, we investigated whether the deposition parameters of SF nanospheres also followed the Hamaker equation in a similar manner to EPD of polymer molecules, which predicts a linear increase in deposited mass with 
a
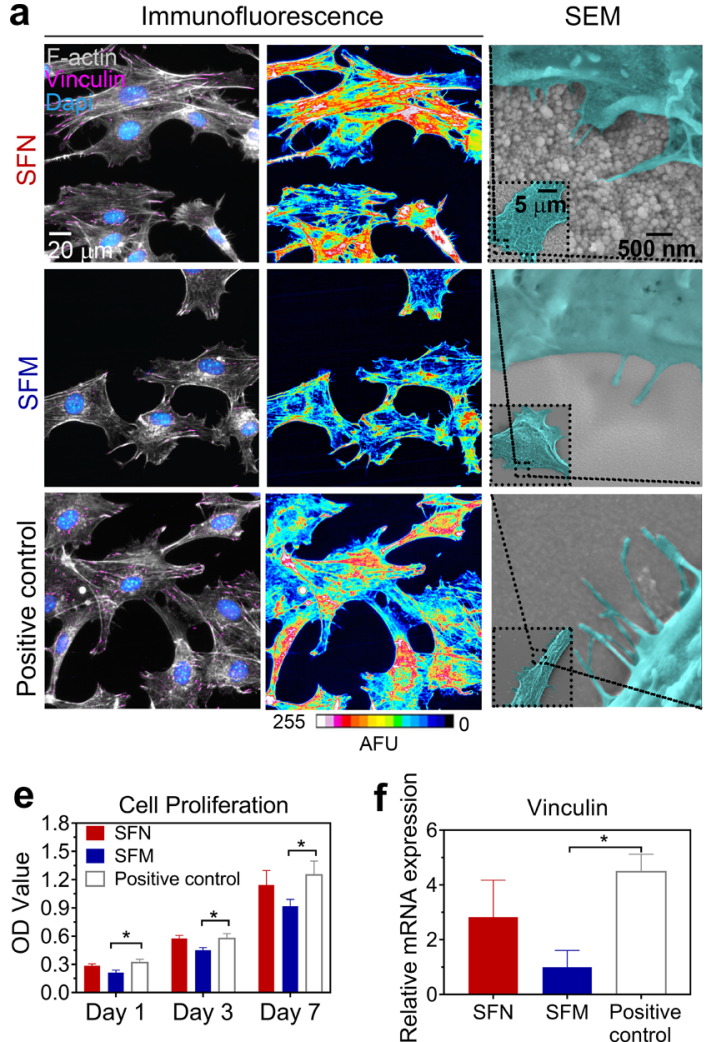
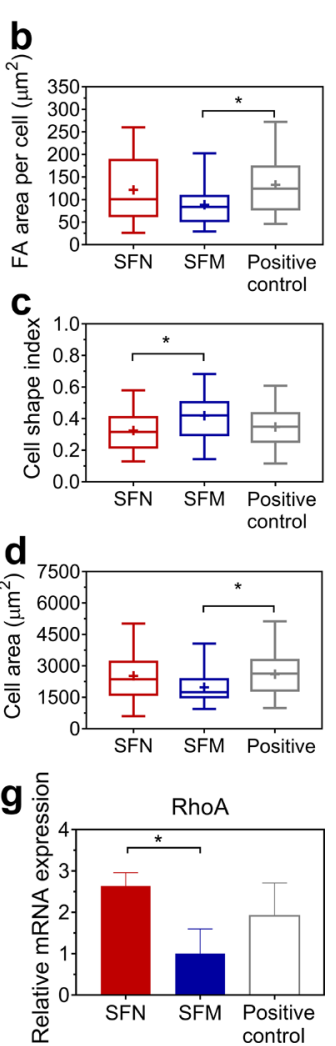

Figure 4. Cellular response to SFN coating. (a) Cell spreading after 24 h shown as immunofluorescent images of vinculin (purple), F-actin (white), and nucleus (blue), corresponding heatmap, and scanning electron micrographs. Quantitative analysis of (b) FA area per cell, (c) CSI, and (d) cell area. (e) Cell proliferation measured by CCK-8. Relative mRNA expression level of (f) vinculin and (g) RhoA. For each box plot, the box boundaries represent the $25-75 \%$ quartiles, and the whiskers represent the minimum and maximum value. Error bars represent one standard deviation $(* p<0.05)$.

increased suspension concentration, electric field, and deposition time. ${ }^{6}$

Figure $2 \mathrm{a}$ revealed the effect of the suspension concentration upon applying a constant electric field of $5 \mathrm{~V} / \mathrm{cm}$ for $2 \mathrm{~min}$. At concentrations below $0.5 \mathrm{wt} \%$, coatings were not deposited, which may have resulted from the fact that the low concentration of nanospheres prevents aggregation. ${ }^{31}$ At concentrations higher than 1.0 wt \%, coating thickness increased linearly with increasing concentration. However, suspension stability decreased with increasing concentration, which complicated dispersion of the suspension. ${ }^{38}$ Therefore, a moderate SF nanosphere concentration of $1.0 \mathrm{wt} \%$ was selected for further studies.

From Figure $2 b$, it can be concluded that the deposition rate increased with an increasing electric field. At electric fields below $3 \mathrm{~V} / \mathrm{cm}$, coatings could not form, which may contribute to the fact that the $\mathrm{pH}$ near the metal substrates was not low enough to cause aggregation and deposition (see eq 5). ${ }^{6}$ However, the SFN coatings became less homogeneous and showed poor attachment to the titanium surface with increasing electric field, which may be caused by the very acute generation of oxygen bubbles at a relatively high electric field (see eq 5). ${ }^{32}$ When the electric field was higher than $8 \mathrm{~V} /$ $\mathrm{cm}$, no deposition was observed anymore. Therefore, a moderate electric field of $5 \mathrm{~V} / \mathrm{cm}$ was selected in further studies to achieve homogeneous coatings.

Figure $2 \mathrm{c}$ reveals that the thickness of the SF nanosphere layer increases linearly when deposition time was increased from 1 to $6 \mathrm{~min}$. This indicates that within $6 \mathrm{~min}$, (i) the EPD process is Faradaic and (ii) the coating thickness can be linearly controlled by deposition time. ${ }^{31}$ However, after $6 \mathrm{~min}$, the process showed self-limitation, as no increase in coating thickness occurred with further prolongation of the coating time. Oxidation of water takes place at the metal/electrolyte interface, whereas deposition occurs at the SF nanosphere layer/electrolyte interface. Consequently, this self-limiting phenomenon coating thickness after 6 min may result from the fact that the distance between these two interfaces increases over time. ${ }^{18}$

3.3. Material Characterization of SFN Coating. Figure 3a shows a typical morphology of an SFN and the conventional SFM coating obtained at comparable conditions. The SFN coating was uniform and revealed a nanostructure resembling the shape of the initial SF nanospheres. The tilted view of the coating interior showed that both SFN and SFM coatings were assembled densely and homogeneously without visible pores.

FTIR results helped us understand the conformational change of SF molecules during coating assembly. For conventional SFM coating, SF molecules first deposit and formed a gel at positive electrodes (Figure 1a) because of the local conformational changes from a random coil to helical state (Figure $3 \mathrm{~b}) .^{39}$ Then, this metastable form of SF was then converted to the more stable crystalline b-sheet structures (Figure $3 b$ ) during the air-dry process which transforms the silk gel to a coating (Figure 1a). At last, water annealing was applied to further induce $\beta$-sheet formation inside coating, which ensures that coating was both chemically stable and water-insoluble. 5 
Scheme 1. Schematic Illustrating Mechanisms of Drug Loading and Release from (a) Pre-Assembled SFNV Coatings vs (b) Conventional SFMV Coatings

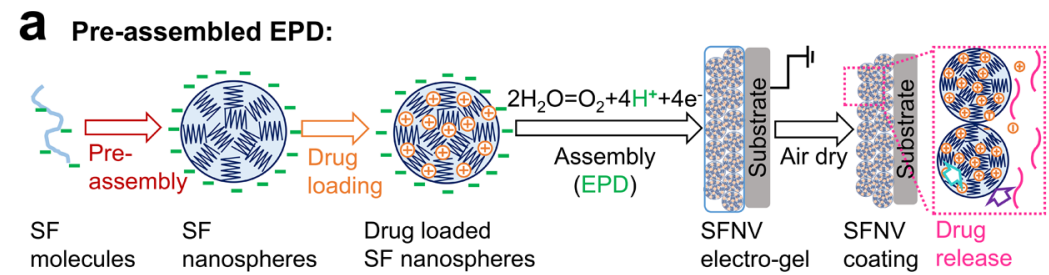

b Conventional EPD:

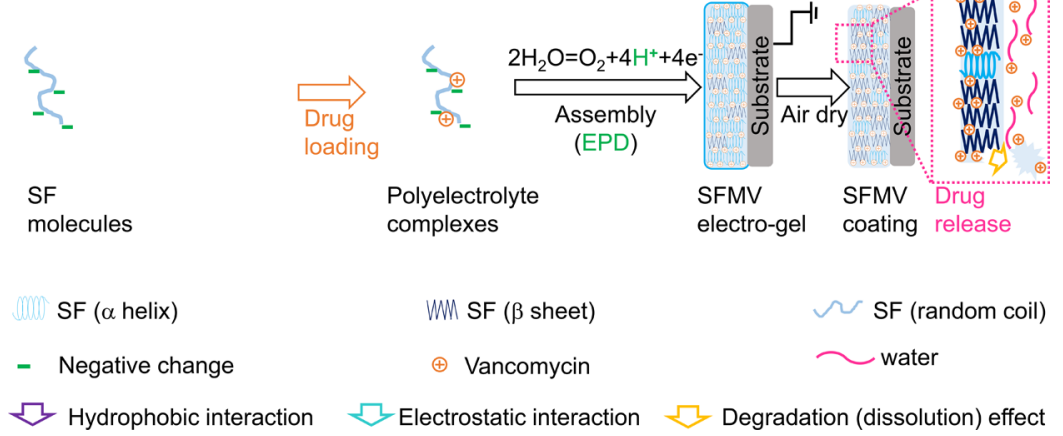

For the SFN coatings, SF nanospheres were first assembled (Figure 1b), which showed a high amount of $\beta$-sheet formation (Figure $3 \mathrm{~b}$ ). This was attributed to exposure of SF molecules to acetone during the pre-assembly process, which dehydrated the SF and facilitated closer chain packing of the hydrophobic Gly-X repeats. ${ }^{30}$ In contrast to the conventional SFM coatings, the conformation of SF was stable and almost unchanged (Figure 3b) immediately after EPD and subsequent drying and annealing of SFN coatings (Figure $1 \mathrm{~b}$ ). This indicates that the annealing step can be omitted for SFN coatings.

The water contact angle results showed that SFN coatings were less hydrophilic than conventional SFM coatings (Figure $3 \mathrm{~d}$ ), which may be caused by the rougher topography (Figure $3 c)$ and the increased hydrophobic $\beta$-sheets in SFN coatings. ${ }^{32}$ In PBS, both coatings showed very limited degradation (Figures $3 \mathrm{e}$ and S2, Supporting Information), which was consistent with a previous study. ${ }^{40}$ However, there was a noticeable weight loss in SFM coating during the first $24 \mathrm{~h}$, which is due to the fact that soluble peptides of the SF leached out into PBS. ${ }^{40,41}$ In contrast, the SFN coating showed no initial weight loss, indicating that the washing step during nanosphere preparation did already remove the soluble components. $^{36}$

The adhesion strength between the coatings and the metallic substrates was investigated in a lap shear tensile test (Figures $3 \mathrm{f}$ and S1, Supporting Information). It could be seen that SFN and SFM coatings exhibited an adhesion strength of $6.66 \pm$ 1.10 and $8.16 \pm 1.31 \mathrm{MPa}$, respectively. However, the change of nanostructures of the SF EPD coatings had no significant influence on adhesion strengths. The strength values observed in this study were similar to or higher than the adhesion strengths of polymer EPD coatings that were reported before being measured by lap shear tensile test (in the range from 1.5 to $8 \mathrm{MPa}) .{ }^{19,42,43}$ Moreover, the coating constructed on the transcutaneous part of implants does not need to resist high mechanical force such as the ones during press or screw-fit placement. ${ }^{12}$ Consequently, we assume that the adhesion strength of coatings was sufficient for our application in this study.

In addition, we anticipate that this novel nanospheres EPD coating might be used on various types of metallic medical implants, considering its feasibility of preparation on different metallic surfaces (Figure S3, Supporting Information).

3.4. Biological Assessment of SFN Coating. Fibroblasts were cultured to clarify the cellular responses to these coatings. The commercial cell culture coverslips were used as the positive control. From fluorescent staining of F-actin and vinculin (Figure 4a), we observed more abundant cytoskeleton organization and FA formation on SFN coating than on SFM coating, which was consistent with more filopodia formation on SFN coating than on SFM coating showed by scanning electron micrographs (Figure 4a). The morphology of the cells on SFN coating tended to be more elongated (Figure 4c). The cell area (Figure 4d), the FA area per cell (Figure 4b), proliferation activity (Figure 4e), and vinculin gene expression (Figure 4f) on SFM coating were smaller than those on the positive control, while these values on SFN coating exhibited no significant difference compared with these values on the positive control. This finding was supported by the upregulated gene expression of RhoA (Figure $4 \mathrm{~g}$ ) in the cell on SFN surfaces compared with SFM. Previous studies demonstrated that via linking F-actin to the exposed cryptic binding sites of unfolded vinculin at FAs, vinculin triggers a series of phosphorylation events to activate the mechanoresponsive signaling transforming protein, RhoA, which engages in the controls of cytoskeleton dynamics and cell polarity. ${ }^{4,45}$

Previous studies also observed that adhesion, spreading, and proliferation of fibroblasts were less on flat silk films than on the flat tissue culture plate. ${ }^{46}$ However, this cellular response could be improved by creating surface roughness by incorporating (diameter of $100 \mathrm{~nm})^{47}$ or establishing porosity (diameter of $80 \mathrm{~nm}),{ }^{48}$ whose feature sizes are comparable to those of our SFN coating. The other surface parameter which might influence cell behavior is hydrophobicity. The water contact angle of SFN coating increased from 60 to $76^{\circ}$ 
a

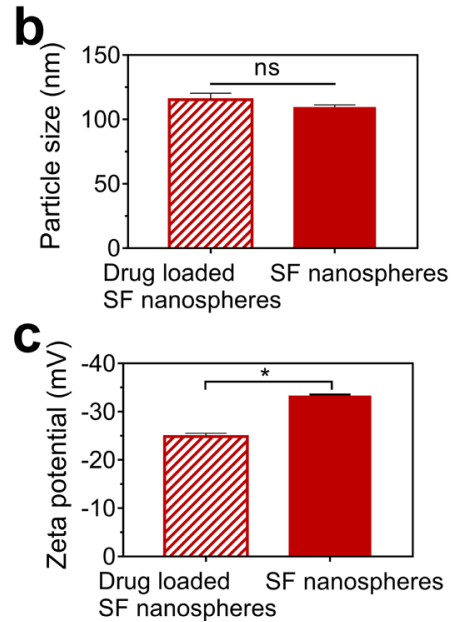

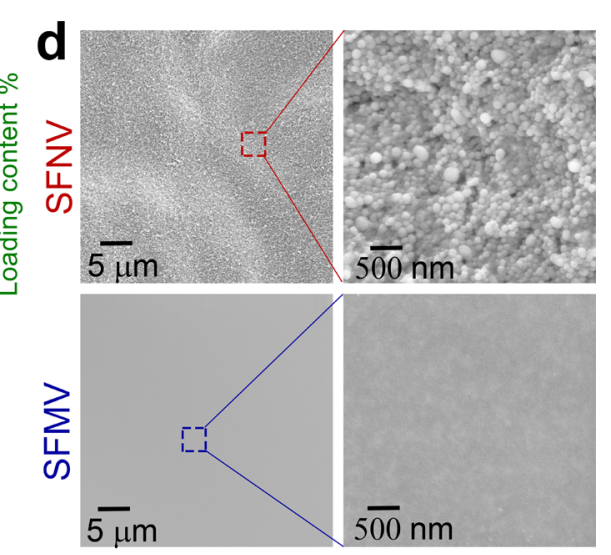

e

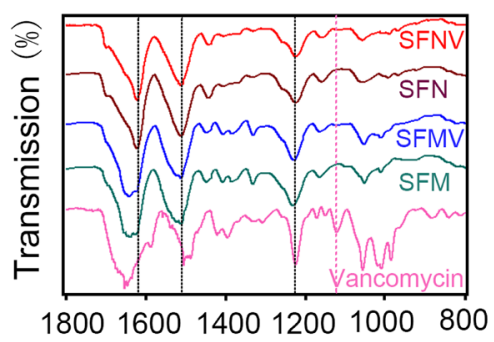

Figure 5. Drug loading onto SFN coating. (a) Encapsulation efficiency and loading content of SF nanospheres as a function of the weight ratio. (b) Particle size and (c) $\zeta$-potential and of SF nanospheres and drug-loaded SF nanospheres. (d) Scanning electron micrographs of SFNV and SFMV coatings. (e) FTIR spectra showing pure vancomycin powder, SFM, SFV, SFN, and SFNV coatings. Error bars represent one standard deviation $(* p<0.05)$.

compared to SFM coatings. However, it has been reported that the fibroblastic cell behavior on surfaces with the water contact angles within this range showed no significant difference, and these moderate hydrophilic material surfaces have the best fibroblastic attachment and spreading compared to more hydrophilic or hydrophobic surfaces. ${ }^{49}$ In summary, the SFN coating enhanced initial fibroblastic responses compared to the conventional SFM coating, which might be helpful for early transcutaneous wound healing and could favor preventing infections. ${ }^{5,50}$

3.5. Drug Loading onto SFN Coating. As a following step, vancomycin was chosen as a model drug to test our hypothesis that SFN coating provides more control over drug release kinetics than conventional SFM coating. Vancomycin was selected because this antibiotic is one of the most widely used antibiotics in the orthopedic surgery to prevent implantassociated infections. ${ }^{11}$ The conventional approach of loading drugs onto the EPD coatings involves blending the drugs with the EPD polymer precursor solution followed by deposition of the mixed solution. ${ }^{6}$ To mimic this conventional drug-loading strategy, vancomycin was first mixed with the SF solution in our study (Scheme 1a). Consequently, the positively charged drug and the negatively charged SF molecules formed polyelectrolyte complexes in the solution. ${ }^{5}$

For our new EPD coating strategy, we first loaded vancomycin onto the SF nanospheres using a diffusional postloading method (Scheme 1b). Our data showed that the drug encapsulation efficiency of SF nanospheres decreased with increasing vancomycin content, while the drug loading capacity of nanospheres first increased and then slightly decreased, showing an inflection point at $12 \% \mathrm{w} / \mathrm{w}$ (vancomycin/SFNs) (Figure 5a). Hence, we selected $12 \%$ $\mathrm{w} / \mathrm{w}$ as the fixed amount of drug loading into the nanospheres, where a maximum loading capacity was reached at $8.3 \%$ with the encapsulation efficiency of $69.3 \%$. When vancomycin was loaded at this ratio, the particle size of the nanospheres (Figure $5 \mathrm{~b}$ ) and the $\mathrm{pH}$ of the suspension remained almost unchanged $(\sim 7)$, whereas the $\zeta$-potential of the nanospheres decreased from -30 to $-20 \mathrm{mV}$ (Figure $5 \mathrm{c}$ ). This decrease of $\zeta$-potential was attributed to partial compensation of the negative charge of SF by the positively charged vancomycin molecules, indicating that electrostatic interactions might be formed between the anionic groups of SF and cationic groups of vancomycin. $^{30}$

Next, we prepared coatings from the vancomycin-loaded SF nanospheres (SFNV coatings) or the vancomycin-loaded SF molecule solution (SFMV coatings), as shown in Scheme 1. For comparison, we used the same vancomycin/protein ratio $(12 \% \mathrm{w} / \mathrm{w})$ and comparable EPD parameters for both coatings. Both coatings showed no significant difference in thickness (Figure S4). Scanning electron micrographs demonstrated that both coatings were deposited feasibly (Figure 5d). The FTIR results (Figure 5e) showed that SFM and SFMV coatings both revealed identical absorbance bands of amide I at $1624 \mathrm{~cm}^{-1}$, amide II at $1513 \mathrm{~cm}^{-1}$, and amide III at 1232 $\mathrm{cm}^{-128}$. Similarly, these three identical characteristic bands were observed at 1619,1512 , and $1228 \mathrm{~cm}^{-1}$ in both SFN and SFNV spectra, respectively. In addition, pure vancomycin, SFMV, and SFNV coatings exhibited an additional identical absorbance band at $1124 \mathrm{~cm}^{-1}$, indicating that vancomycin was successfully loaded into both SFNV and SFMV coatings. Furthermore, no distinct new bands or peak shifts were observed, suggesting that no extra chemical reactions or formation of covalent bonds occurred between SF and 
vancomycin during EPD. ${ }^{5}$ Moreover, the amide I spectra of SFNV (SFMV) coatings (Figure S5, Supporting Information) show similar conformational changes during the preparation process as observed for SFN (SFM) coatings (Figure 3b), indicating that SFNV (SFMV) coating was assembled by the similar principles as SFN (SFM) coatings as discussed above (Scheme 1 and Figure 1a,b).

3.6. Drug Release from SFN Coating. Subsequently, the release of vancomycin from SFNV and SFMV coatings in PBS was monitored by HPLC. The maximum release amount of vancomycin was $38 \%$ higher $(p<0.05)$ from SFNV coating than from SFMV coating (Figure 6a). In addition, burst release
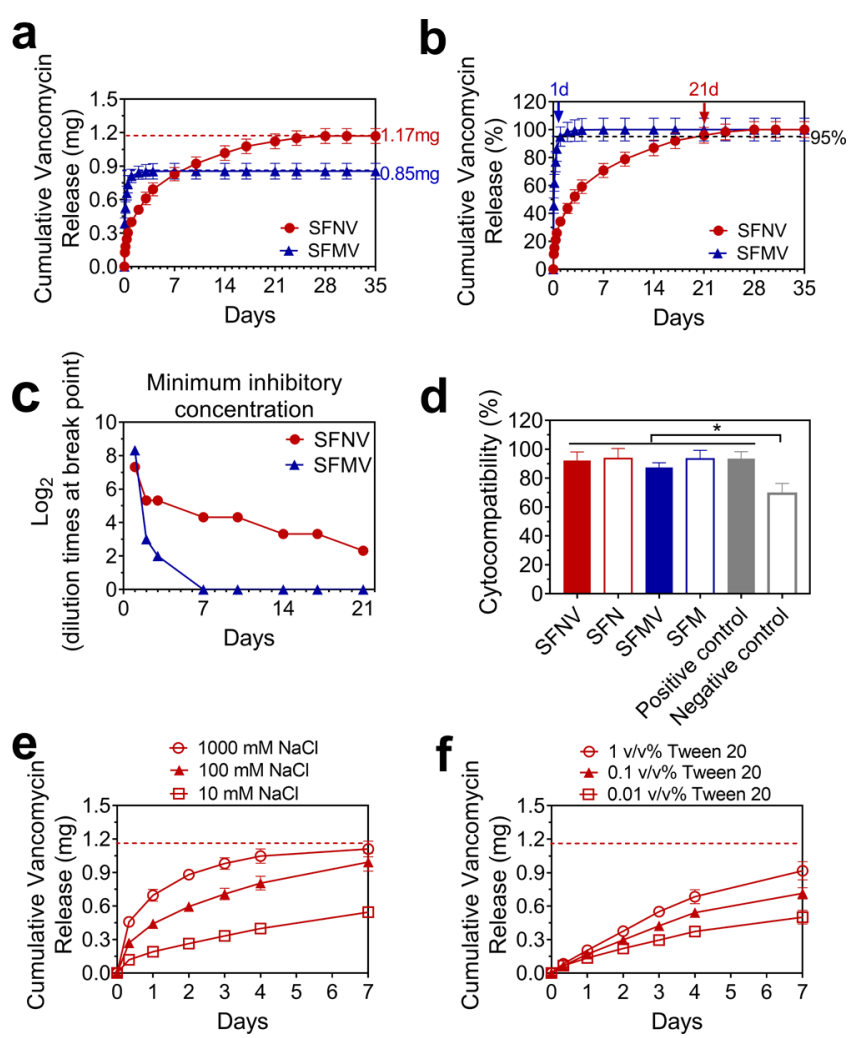

Figure 6. Drug Release from SFN coating. (a) Vancomycin release profiles shown as the cumulative release amount, and the dashed line indicating the maximum release amount. (b) Vancomycin release profiles shown as a cumulative release percentage and the dashed line indicating 95\% maximum release amount with arrows indicating when it arrives. (c) MIC tests showing the antibacterial bioactivity of SFNV and SFMV coatings at different time points. (d) Cytocompatibility of SFNV and SFMV coatings. Vancomycin release kinetics from SFN coatings in media of different (e) ionic strength and (f) detergent concentrations. Error bars represent one standard deviation $(* p<$ $0.05)$.

of 95\% vancomycin from SFMV coatings was observed within 1 day (Figure 6b). In contrast, the sustained release of vancomycin was observed on SFNV coating, and 95\% maximum release amount of vancomycin was released for 21 days (Figure 6b). Moreover, the physiological environment surrounding implants sometimes can experience a decrease in $\mathrm{pH}$ because of the infection, and previous studies have demonstrated that this $\mathrm{pH}$ decrease can further extend the release time of vancomycin from SF nanospheres. ${ }^{30}$ These results confirmed that assembly of EPD coatings from nanospheres not only prolonged drug delivery kinetics but also enhanced the drug release amount considerably.

Furthermore, to examine the effectiveness of released vancomycin of both EPD coatings, $S$. aureus, one of the most common pathogenic bacteria in infections associated with surgical implants, ${ }^{20}$ was used for our antibacterial tests. The minimum inhibition concentration (MIC) test confirmed the effective drug bioactivity of released vancomycin from SFNV coatings against $S$. aureus for at least 21 days. In contrast, the released drug from SFMV coatings only evoked an antibacterial effect during the first 3 days (Figure 6c). In addition, both of the drug-loaded coatings were not cytotoxic to fibroblasts (Figure 6d).

The first-order, Higuchi, and Korsmeyer-Peppas models were used fitted to the release data of SFNV and SFMV coatings (Figure S6). The correlation coefficients were 0.980.99, 0.91-0.97, and 0.95-0.99 for the first-order, Higuchi, and Korsmeyer-Peppas models, respectively. The correlation coefficients and visual inspection of the plots showed that the drug release kinetics from SFNV and SFMV coatings can fit with all three models, but the kinetics were more close to the first-order model release than the others. Furthermore, the values of $n(0.29-0.33)$ of both SF coatings in KorsmeyerPeppas models were smaller than the critical value of 0.45 . This suggests that vancomycin in both coatings was released through a Fickian diffusion mechanism. ${ }^{51}$

Finally, we investigated the underlying mechanism by which SFNV coating facilitated sustained delivery of vancomycin over prolonged time periods. SF macromolecules consist of both positively ( $R$ and $K$ ) and negatively charged ( $D$ and $E$ ), hydrophobic (A, G, L, V, W, C, I, M, F, P), and hydrophilic $(\mathrm{N}, \mathrm{Q}, \mathrm{S}, \mathrm{T}, \mathrm{Y}, \mathrm{R}, \mathrm{D}, \mathrm{E}, \mathrm{H}, \mathrm{K})$ amino acids. ${ }^{52}$ This composition of SF enables establishment of electrostatic and hydrophobic interactions between positively charged vancomycin molecules and SF carriers. The electrostatic interactions between vancomycin and SF nanospheres (Scheme 1a) were confirmed by the fact that vancomycin release was enhanced with an increase in the ionic strength of the release medium (Figure $6 \mathrm{e})$. The electric double layer of the nanospheres was compressed with increasing ionic strength. ${ }^{53,54}$ Consequently, positively charged sodium ions entered the Stern layer of nanospheres and competed with vancomycin molecules to form stable complexes, ${ }^{54}$ which resulted in an accelerated release of vancomycin.

During the pre-assembly process of SF nanospheres, acetone dehydrates SF and leads to the inward folding of the hydrophobic part of SF molecules. ${ }^{36}$ To confirm the formation of hydrophobic interactions between the hydrophobic group of vancomycin and SF nanospheres, detergent Tween 20 was added into the release media because amphiphilic Tween 20 can disrupt such hydrophobic interactions. ${ }^{54}$ The moderate enhancement of vancomycin release with increasing Tween 20 concentration (Figure 6f) was a clear indication that hydrophobic interactions were formed as well between vancomycin and SF nanospheres (Scheme 1a).

In addition to the electrostatic and hydrophobic interactions, less initial dissolution (degradation) of the nanosphere coatings was observed within the first $24 \mathrm{~h}$ as discussed above (Figure 3e). This may also play an essential role in minimizing the burst release of vancomycin from SFN compared to conventional SFM coatings (Scheme $1 \mathrm{~b}$ ).

Although we have only focused on SF nanoparticles here, other polymer precursors used in EPD such as chitosan and 
alginate, have also been intensely investigated to assemble various types of nanovehicles for control release of drugs for biomedical applications. ${ }^{55-57}$ We envision that this developed strategy might be applied to those materials to tailor drug release from EPD coatings to meet application-specific needs with using no (or as less as possible) additives. Moreover, this novel EPD strategy might also provide a possibility to tune drug release rate within a wide range by assembling the coatings from different ratios of molecules and pre-assembled nanostructures to meet various clinical applications in future.

\section{CONCLUSIONS}

We put forward a simple, green, and economic pre-assembly strategy to improve the drug release of polymer EPD coating without introducing any additives except the polymer precursor itself. This feasibility of this concept was demonstrated by developing a novel SF EPD coating assembled from SF nanospheres to improve the delivery drug in an application model, that is, preventing infections around percutaneous orthopedic implants via local delivery of antibiotics. The proposed EPD mechanism of SFN coating involved oxidation of water near the substrate to neutralize SF nanospheres resulting in irreversible deposition. The deposition process and mass could be easily controlled using the applied EPD parameters. Compared to conventional SFM coating, this nanostructured SFN coating had a more rough, more hydrophobic, and more stable surface with better cell response and similar adhesion strength. Most importantly, the use of nanospheres as building blocks enhanced 1.38 times on the maximum drug release amount and prolonged 21 times on drug release time (95\% maximum release) while retaining drug effectiveness without detectable cytotoxicity. This superior release form SFN coatings resulted from the electrostatic and hydrophobic interactions between the drug and nanospheres, and less initial dissolution effect on nanosphere coating. These results illustrate the potential of the pre-assembly strategy on EPD polymer coatings used for drug-delivery applications.

\section{ASSOCIATED CONTENT}

\section{SI Supporting Information}

The Supporting Information is available free of charge at https://pubs.acs.org/doi/10.1021/acsami.9b21808.

Lap shear tensile test for adhesion strength of coatings; degradation of coatings; feasibility of constructing SFN coatings on different typical medical metals; thickness of coatings loaded with and without vancomycin; FTIR absorbance spectra of the amide I region showing the SF conformation change during the preparation of SFNV and SFMV coatings; fits of the first-order, Higuchi, and Korsmeyer-Peppas model equations to the drug release data; primer sets (PDF)

\section{AUTHOR INFORMATION}

\section{Corresponding Author}

Fang Yang - Department of Dentistry-Biomaterials, Radboud University Medical Center, Nijmegen 6525 EX, The

Netherlands; 10 orcid.org/0000-0002-4022-7643;

Email: Fang.Yang@radboudumc.nl

\section{Authors}

Xian Cheng - Department of Dentistry-Biomaterials, Radboud University Medical Center, Nijmegen 6525 EX, The Netherlands

Dongmei Deng - Department of Preventive Dentistry, Academic Center for Dentistry Amsterdam, University of Amsterdam and Vrije Universiteit Amsterdam, Amsterdam 1081, The Netherlands

Lili Chen - Department of Stomatology, Union Hospital, Tongji Medical College, Huazhong University of Science and Technology, Wuhan 430022, P. R. China

John A. Jansen - Department of Dentistry-Biomaterials, Radboud University Medical Center, Nijmegen 6525 EX, The Netherlands

Sander G. C. Leeuwenburgh - Department of DentistryBiomaterials, Radboud University Medical Center, Nijmegen 6525 EX, The Netherlands; (1) orcid.org/0000-0003-14716133

Complete contact information is available at:

https://pubs.acs.org/10.1021/acsami.9b21808

\section{Notes}

The authors declare no competing financial interest.

\section{ACKNOWLEDGMENTS}

X.C. is funded by China Scholarship Council (project no. 201606160095). X.C. would like to thank Jinmeng Li, Kaiwen Chen, Yanping Liu, Jiamian Wang, and Dr Jinglong Shao for helpful discussions and suggestions. Silk cocoons are kindly provided by Prof. Aichun Zhao (State Key Laboratory of Silkworm Genome Biology, Southwest University, Chongqing, P. R. China).

\section{REFERENCES}

(1) Burt, H. M.; Hunter, W. L. Drug-eluting stents: A Multidisciplinary Success Story. Adv. Drug Delivery Rev. 2006, 58, 350-357.

(2) Wang, Y.; Zhang, W.; Zhang, J.; Sun, W.; Zhang, R.; Gu, H. Fabrication of a Novel Polymer-Free Nanostructured Drug-Eluting Coating for Cardiovascular Stents. ACS Appl. Mater. Interfaces 2013, 5, 10337-10345.

(3) Goodman, S. B.; Yao, Z.; Keeney, M.; Yang, F. The Future of Biologic Coatings for Orthopaedic Implants. Biomaterials 2013, 34, 3174-3183.

(4) Tobin, E. J. Recent Coating Developments for Combination Devices in Orthopedic and Dental Applications: A Literature Review. Adv. Drug Delivery Rev. 2017, 112, 88-100.

(5) Han, C.; Yao, Y.; Cheng, X.; Luo, J.; Luo, P.; Wang, Q.; Yang, F.; Wei, Q.; Zhang, Z. Electrophoretic Deposition of Gentamicin-loaded Silk Fibroin Coatings on 3D-printed Porous Cobalt-ChromiumMolybdenum Bone Substitutes to Prevent Orthopedic Implant Infections. Biomacromolecules 2017, 18, 3776-3787.

(6) Avcu, E.; Baştan, F. E.; Abdullah, H. Z.; Rehman, M. A. U.; Avcu, Y. Y.; Boccaccini, A. R. Electrophoretic Deposition of Chitosan-Based Composite Coatings for Biomedical Applications: A review. Prog. Mater. Sci. 2019, 103, 69-108.

(7) Sun, J.; Zhu, Y.; Meng, L.; Chen, P.; Shi, T.; Liu, X.; Zheng, Y. Electrophoretic Deposition of Colloidal Particles on $\mathrm{Mg}$ with Cytocompatibility, Antibacterial Performance, and Corrosion Resistance. Acta Biomater. 2016, 45, 387-398.

(8) Lei, M.; Qu, X.; Liu, H.; Liu, Y.; Wang, S.; Wu, S.; Bentley, W. E.; Payne, G. F.; Liu, C. Programmable Electrofabrication of Porous Janus Films with Tunable Janus Balance for Anisotropic Cell Guidance and Tissue Regeneration. Adv. Funct. Mater. 2019, 29, 1900065. 
(9) Qi, H.; Heise, S.; Zhou, J.; Schuhladen, K.; Yang, Y.; Cui, N.; Dong, R.; Virtanen, S.; Chen, Q.; Boccaccini, A. R.; Lu, T. Electrophoretic Deposition of Bioadaptive Drug Delivery Coatings on Magnesium Alloy for Bone Repair. ACS Appl. Mater. Interfaces 2019, 11, 8625-8634.

(10) Diba, M.; Fam, D. W. H.; Boccaccini, A. R.; Shaffer, M. S. P. Electrophoretic Deposition of Graphene-Related Materials: A Review of the Fundamentals. Prog. Mater. Sci. 2016, 82, 83-117.

(11) Bakhshandeh, S.; Gorgin Karaji, Z.; Lietaert, K.; Fluit, A. C.; Boel, C. H. E.; Vogely, H. C.; Vermonden, T.; Hennink, W. E.; Weinans, H.; Zadpoor, A. A.; Amin Yavari, S. Simultaneous Delivery of Multiple Antibacterial Agents from Additively Manufactured Porous Biomaterials to Fully Eradicate Planktonic and Adherent Staphylococcus aureus. ACS Appl. Mater. Interfaces 2017, 9, 2569125699.

(12) Song, J.; Chen, Q.; Zhang, Y.; Diba, M.; Kolwijck, E.; Shao, J.; Jansen, J. A.; Yang, F.; Boccaccini, A. R.; Leeuwenburgh, S. C. G. Electrophoretic Deposition of Chitosan Coatings Modified with Gelatin Nanospheres To Tune the Release of Antibiotics. ACS Appl. Mater. Interfaces 2016, 8, 13785-13792.

(13) Patel, K. D.; Kim, T.-H.; Lee, E.-J.; Han, C.-M.; Lee, J.-Y.; Singh, R. K.; Kim, H.-W. Nanostructured Bio-interfacing of Metals with Carbon Nanotube / Chitosan Hybrids by Electrodeposition for Cell Stimulation and Therapeutics Delivery. ACS Appl. Mater. Interfaces 2014, 6, 20214.

(14) Ran, J.; Zeng, H.; Cai, J.; Jiang, P.; Yan, P.; Zheng, L.; Bai, Y.; Shen, X.; Shi, B.; Tong, H. Rational Design of a Stable, Effective, and Sustained Dexamethasone Delivery Platform on a Titanium Implant: An Innovative Application Of Metal Organic Frameworks In Bone Implants. Chem. Eng. J. 2018, 333, 20-33.

(15) Chen, R.; Cai, X.; Ma, K.; Zhou, Y.; Wang, Y.; Jiang, T. The Fabrication of Double-Layered Chitosan/Gelatin/Genipin Nanosphere Coating for Sequential and Controlled Release of Therapeutic Proteins. Biofabrication 2017, 9, 025028.

(16) Raphel, J.; Holodniy, M.; Goodman, S. B.; Heilshorn, S. C. Multifunctional Coatings to Simultaneously Promote Osseointegration and Prevent Infection of Orthopaedic Implants. Biomaterials 2016, 84, 301-314.

(17) Mottaghitalab, F.; Farokhi, M.; Shokrgozar, M. A.; Atyabi, F.; Hosseinkhani, H. Silk Fibroin Nanoparticle as a Novel Drug Ddelivery System. J. Controlled Release 2015, 206, 161-176.

(18) Pishbin, F.; Mouriño, V.; Gilchrist, J. B.; McComb, D. W.; Kreppel, S.; Salih, V.; Ryan, M. P.; Boccaccini, A. R. Single-Step Electrochemical Deposition of Antimicrobial Orthopaedic Coatings Based on A Bioactive Glass/Chitosan/Nano-Silver Composite System. Acta Biomater. 2013, 9, 7469-7479.

(19) Zhang, Z.; Qu, Y.; Li, X.; Zhang, S.; Wei, Q.; Shi, Y.; Chen, L. Electrophoretic Deposition of Tetracycline Modified Silk Fibroin Coatings for Functionlization of Titanium Surfaces. Appl. Surf. Sci. 2014, 303, 255-262.

(20) Croes, M.; Bakhshandeh, S.; van Hengel, I. A. J.; Lietaert, K.; van Kessel, K. P. M.; Pouran, B.; van der Wal, B. C. H.; Vogely, H. C.; Van Hecke, W.; Fluit, A. C.; Boel, C. H. E.; Alblas, J.; Zadpoor, A. A.; Weinans, H.; Amin Yavari, S. Antibacterial and Immunogenic Behavior of Silver Coatings on Additively Manufactured Porous Titanium. Acta Biomater. 2018, 81, 315-327.

(21) Elia, R.; Michelson, C. D.; Perera, A. L.; Brunner, T. F.; Harsono, M.; Leisk, G. G.; Kugel, G.; Kaplan, D. L. Electrodeposited Silk Coatings for Bone Implants. Biomed. Mater. Res., Part B 2015, 103, 1602-1609.

(22) Bressner, J. E.; Marelli, B.; Qin, G.; Klinker, L. E.; Zhang, Y.; Kaplan, D. L.; Omenetto, F. G. Rapid Fabrication of Silk Films with Controlled Architectures via Electrogelation. J. Mater. Chem. B 2014, 2, 4983-4987.

(23) Leisk, G. G.; Lo, T. J.; Yucel, T.; Lu, Q.; Kaplan, D. L. Electrogelation For Protein Adhesives. Adv. Mater. 2010, 22, 711715 .
(24) Yucel, T.; Lovett, M. L.; Kaplan, D. L. Silk-Based Biomaterials for Sustained Drug Delivery. J. Controlled Release 2014, 190, 381397.

(25) Kundu, B.; Rajkhowa, R.; Kundu, S. C.; Wang, X. Silk Fibroin Biomaterials for Tissue Regenerations. Adv. Drug Delivery Rev. 2013, 65, 457-470.

(26) Numata, K.; Kaplan, D. L. Silk-Based Delivery Systems of Bioactive Molecules. Adv. Drug Delivery Rev. 2010, 62, 1497-1508.

(27) Mathur, A. B.; Gupta, V. Silk Fibroin-Derived Nanoparticles For Biomedical Applications. Nanomedicine 2010, 5, 807-820.

(28) Tian, Y.; Jiang, X.; Chen, X.; Shao, Z.; Yang, W. DoxorubicinLoaded Magnetic Silk Fibroin Nanoparticles for Targeted Therapy of Multidrug-Resistant Cancer. Adv. Mater. 2014, 26, 7393-7398.

(29) Rockwood, D. N.; Preda, R. C.; Yücel, T.; Wang, X.; Lovett, M. L.; Kaplan, D. L. Materials fabrication from Bombyx mori silk fibroin. Nat. Protoc. 2011, 6, 1612.

(30) Hassani Besheli, N.; Mottaghitalab, F.; Eslami, M.; Gholami, M.; Kundu, S. C.; Kaplan, D. L.; Farokhi, M. Sustainable Release of Vancomycin from Silk Fibroin Nanoparticles for Treating Severe Bone Infection in Rat Tibia Osteomyelitis Model. ACS Appl. Mater. Interfaces 2017, 9, 5128-5138.

(31) Geuli, O.; Metoki, N.; Eliaz, N.; Mandler, D. Electrochemically Driven Hydroxyapatite Nanoparticles Coating of Medical Implants. Adv. Funct. Mater. 2016, 26, 8003-8010.

(32) Zhang, Z.; Cheng, X.; Yao, Y.; Luo, J.; Tang, Q.; Wu, H.; Lin, S.; Han, C.; Wei, Q.; Chen, L. Electrophoretic Deposition of Chitosan/Gelatin Coatings with Controlled Porous Surface Topography to Enhance Initial Osteoblast Adhesive Responses. J. Mater. Chem. B 2016, 4, 7584-7595.

(33) Diba, M.; García-Gallastegui, A.; Klupp Taylor, R. N.; Pishbin, F.; Ryan, M. P.; Shaffer, M. S. P.; Boccaccini, A. R. Quantitative Evaluation of Electrophoretic Deposition Kinetics of Graphene Oxide. Carbon 2014, 67, 656-661.

(34) Horzum, U.; Ozdil, B.; Pesen-Okvur, D. Step-by-step Quantitative Analysis of Focal Adhesions. MethodsX 2014, 1, 56-59.

(35) Caliari, S. R.; Vega, S. L.; Kwon, M.; Soulas, E. M.; Burdick, J. A. Dimensionality and Spreading Influence MSC YAP/TAZ Signaling in Hydrogel Environments. Biomaterials 2016, 103, 314-323.

(36) Seib, F. P.; Jones, G. T.; Rnjak-Kovacina, J.; Lin, Y.; Kaplan, D. L. pH-Dependent Anticancer Drug Release from Silk Nanoparticles. Adv. Healthcare Mater. 2013, 2, 1606-1611.

(37) Kojic, N.; Panzer, M. J.; Leisk, G. G.; Raja, W. K.; Kojic, M.; Kaplan, D. L. Ion Electrodiffusion Governs Silk Electrogelation. Soft Matter 2012, 8, 6897-6905.

(38) Jo, Y. K.; Choi, B. H.; Kim, C. S.; Cha, H. J. Diatom-Inspired Silica Nanostructure Coatings with Controllable Microroughness Using an Engineered Mussel Protein Glue to Accelerate Bone Growth on Titanium-Based Implants. Adv. Mater. 2017, 29, 1704906.

(39) Lu, Q.; Huang, Y.; Li, M.; Zuo, B.; Lu, S.; Wang, J.; Zhu, H.; Kaplan, D. L. Silk Fibroin Electrogelation Mechanisms. Acta Biomater. 2011, 7, 2394-2400.

(40) Li, M.; Ogiso, M.; Minoura, N. Enzymatic degradation behavior of porous silk fibroin sheets. Biomaterials 2003, 24, 357-365.

(41) Brown, J.; Lu, C.-L.; Coburn, J.; Kaplan, D. L. Impact of Silk Biomaterial Structure on Proteolysis. Acta Biomater. 2015, 11, 212221.

(42) Jiang, T.; Zhang, Z.; Zhou, Y.; Liu, Y.; Wang, Z.; Tong, H.; Shen, X.; Wang, Y. Surface Functionalization of Titanium with Chitosan/Gelatin via Electrophoretic Deposition: Characterization and Cell Behavior. Biomacromolecules 2010, 11, 1254-1260.

(43) Zhang, Z.; Jiang, T.; Ma, K.; Cai, X.; Zhou, Y.; Wang, Y. Low Temperature Electrophoretic Deposition of Porous Chitosan/Silk Fibroin Composite Coating for Titanium Biofunctionalization. J. Mater. Chem. 2011, 21, 7705.

(44) Parsons, J. T.; Horwitz, A. R.; Schwartz, M. A. Cell Adhesion: Integrating Cytoskeletal Dynamics and Cellular Tension. Nat. Rev. Mol. Cell Biol. 2010, 11, 633-643.

(45) Carisey, A.; Tsang, R.; Greiner, A. M.; Nijenhuis, N.; Heath, N.; Nazgiewicz, A.; Kemkemer, R.; Derby, B.; Spatz, J.; Ballestrem, C. 
Vinculin Regulates the Recruitment and Release of Core Focal Adhesion Proteins in a Force-Dependent Manner. Curr. Biol. 2013, 23, 271-281.

(46) Wohlrab, S.; Müller, S.; Schmidt, A.; Neubauer, S.; Kessler, H.; Leal-Egaña, A.; Scheibel, T. Cell Adhesion and Proliferation on RGDModified Recombinant Spider Silk Proteins. Biomaterials 2012, 33, 6650-6659.

(47) Lipski, A. M.; Pino, C. J.; Haselton, F. R.; Chen, I.-W.; Shastri, V. P. The Effect of Silica Nanoparticle-Modified Surfaces on Cell Morphology, Cytoskeletal Organization and Function. Biomaterials 2008, 29, 3836-3846.

(48) Nasrollahi, S.; Banerjee, S.; Qayum, B.; Banerjee, P.; Pathak, A. Nanoscale Matrix Topography Influences Microscale Cell Motility through Adhesions, Actin Organization, and Cell Shape. ACS Biomater. Sci. Eng. 2017, 3, 2980-2986.

(49) Kim, S. H.; Ha, H. J.; Ko, Y. K.; Yoon, S. J.; Rhee, J. M.; Kim, M. S.; Lee, H. B.; Khang, G. Correlation of Proliferation, Morphology and Biological Responses of Fibroblasts on LDPE with Different Surface Wettability. J. Biomater. Sci., Polym. Ed. 2007, 18, 609-622.

(50) Jennison, T.; McNally, M.; Pandit, H. Prevention of Infection in External Fixator Pin Sites. Acta Biomater. 2014, 10, 595-603.

(51) Liu, X.; Yang, Y.; Yu, D.-G.; Zhu, M.-J.; Zhao, M.; Williams, G. R. Tunable Zero-Order Drug Delivery Systems Created by Mmodified Triaxial Electrospinning. Chem. Eng. J. 2019, 356, 886894.

(52) Lammel, A. S.; Hu, X.; Park, S.-H.; Kaplan, D. L.; Scheibel, T. R. Controlling Silk Fibroin Particle Features for Drug Delivery. Biomaterials 2010, 31, 4583-4591.

(53) Wang, H.; Hansen, M. B.; Löwik, D. W. P. M.; van Hest, J. C. M.; Li, Y.; Jansen, J. A.; Leeuwenburgh, S. C. G. Oppositely Charged Gelatin Nanospheres as Building Blocks for Injectable and Biodegradable Gels. Adv. Mater. 2011, 23, H119-H124.

(54) Song, J.; Odekerken, J. C. E.; Löwik, D. W. P. M.; López-Pérez, P. M.; Welting, T. J. M.; Yang, F.; Jansen, J. A.; Leeuwenburgh, S. C. G. Influence of the Molecular Weight and Charge of Antibiotics on Their Release Kinetics From Gelatin Nanospheres. Macromol. Biosci. 2015, 15, 901-911.

(55) Elzoghby, A. O. Gelatin-Based nanoparticles as Drug and Gene Delivery Systems: Reviewing Three Decades of Research. J. Controlled Release 2013, 172, 1075-1091.

(56) Shukla, S. K.; Mishra, A. K.; Arotiba, O. A.; Mamba, B. B. Chitosan-Based Nanomaterials: A State-of-the-Art Review. Int. J. Biol. Macromol. 2013, 59, 46-58.

(57) Severino, P.; da Silva, C. F.; Andrade, L. N.; de Lima Oliveira, D.; Campos, J.; Souto, E. B. Alginate Nanoparticles for Drug Delivery and Targeting. Curr. Pharm. Des. 2019, 25, 1312-1334. 